\title{
Social capital and mental health during the COVID-19 pandemic
}

\author{
Tahmina Tasnim Rodela ${ }^{1}$, Abida Sultana ${ }^{2}$, E. Lisako J. Mckyer ${ }^{3}$, \\ Sudip Bhattacharya ${ }^{4}$, Md Mahbub Hossain ${ }^{3 *}$
}

\begin{abstract}
COVID-19 pandemic is affecting mental health and wellbeing across populations. The continued burden of psychosocial stressors in different contexts necessitates multipronged interventions that address mental health problems and associated disparities. Global evidence suggests that social capital plays a critical role in determining mental health outcomes in communities, which can be leveraged for improving mental health in COVID-19. As communities with better social capital is likely to have a lower burden of mental disorders, it is necessary to assess potential measures to use social capital for mental health promotion in vulnerable populations. The existing forms of social capital may provide social support within and between communities that are critically affected by COVID-19. Alongside faster recovery from socioeconomic challenges, social capital interventions may strengthen the social determinants of mental health and empower communities to alleviate the psychosocial consequences of this pandemic.
\end{abstract}

Keywords: Mental Health; Mental Disorders; Social Capital; COVID-19; Coronavirus; Psychosocial Interventions

\footnotetext{
${ }^{1}$ Mawlana Bhashani Science and Technology University, Tangail 1902, Bangladesh.

${ }^{2}$ Gazi Medical College, Khulna 09000, Bangladesh.

${ }^{3}$ School of Public Health, Texas A\&M University, College Station, TX 77843, USA.

${ }^{4}$ Department of Community Medicine, HIMS, Dehradun, India.

*Corresponding author: Md Mahbub Hossain, School of Public Health, Texas A\&M University, College Station, TX 77843, USA. Email: mhossain@tamu.edu
} 


\section{Introduction}

The coronavirus disease (COVID-19) pandemic has critically impacted mental health and wellbeing globally (Hossain et al., 2020b; Rajkumar, 2020). Since the beginning of this pandemic, unpredictability and uncertainty regarding infection prevention, clinical care, and health outcomes have affected psychosocial health across populations. Moreover, preventive strategies like lockdown and physical distancing might have led to social isolation, unemployment, loneliness, and inadequate access to essential commodities, which have impacted psychosocial wellbeing (Hossain et al., 2020b). The mental health impacts of this pandemic are more severe among individuals with existing mental health problems or those who were already exposed to psychological risk factors. As most health systems are poorly equipped with mental health services and resources, there is a growing concern on strengthening mental health through socio-ecological approaches that may alleviate the increasing burden of mental health problems in this pandemic (Hossain et al., 2020a; Rajkumar, 2020; Sentell et al., 2020). Global evidence supports that social capital plays a critical role during public health crises as well as contributes to better mental health in communities and populations. In this article, we briefly discuss social capital and mental health in the context of COVID-19 that may inform psychosocial policymaking and practice in this pandemic.

\section{Social capital and mental health: an overview of the evidence}

Social capital is a well-researched concept that emphasizes social and cognitive constructs like social cohesion, trust, norms, and networks (Ferlander, 2007). Two major forms of social capital, namely bonding capital (social ties within a community) and bridging capital (social ties between communities), may provide critical support that is essential for improving and sustaining social determinants of health (Chen and Meng, 2015; Ferlander, 2007). The fundamental constructs of social capital work together in communities and contribute to collective efficacy leading to better health and wellbeing among individuals. Previous research has explored how social capital is associated with mental health in different contexts. A systematic review of 55 studies found that family and community social capital were associated with behavioral and mental health in children and adolescents (McPherson et al., 2014). Another review of 39 studies reported that individual social capital had protective roles against developing common mental disorders (Ehsan and De 
Silva, 2015). A similar review by De Silva and colleagues evaluated 21 studies and reported that individual and ecological social capital might have an inverse relationship with mental health problems across populations (De Silva et al., 2005). The role of social capital is also examined in vulnerable populations who may have poor psychosocial health and associated risk factors. For example, Nyqvist and colleagues systematically assessed 11 studies that reported positive associations between social capital and mental health in older adults (Nyqvist et al., 2013). While most of the research syntheses highlighted the roles of social capital in improving mental health, Noel and colleagues reviewed 15 studies that evaluated the association between social capital and mental health in public health crises like disasters and conflicts (Noel et al., 2018). This review suggested that cognitive social capital had an inverse association with mental health problems, including post-traumatic stress disorder, anxiety, and depression. The available evidence informs the potentials of social capital for ensuring optimal mental health and wellbeing in normal conditions as well as in crises, which should be examined in the context of the COVID-19 pandemic.

\section{Social capital and COVID-19: implications for mental health}

COVID-19 pandemic has resulted in the closure of public places, whereas lockdown and stay-athome orders have decreased social gatherings and connectedness. These factors may affect social capital as well as mental health among the affected individuals. Nonetheless, the worsening economic indices with no foreseeable end, individual and collective helplessness, negative emotions, and other psychosocial challenges are thriving around the world (Aluh and Onu, 2020). In this scenario, the relationship social capital can be affected in three major ways. First, existing means of social support originating from bonding social capital may not be leveraged effectively during this pandemic, which may affect psychosocial wellbeing in individuals. Second, as most communities remain isolated and disconnected, leveraging bridging social capital remains equally challenging in this scenario, which may further affect the social determinants of mental health. Lastly, institutional efforts for facilitating social capital remain suboptimal as most health systems, clinical and social care providers continue to struggle for preventing the spread of COVID-19 and providing clinical care to infected individuals. Therefore, the use of existing social capital or deployment of social capital interventions from external sources can be inadequate in this 
pandemic, which may impact mental health and wellbeing at individual, community, and population levels.

A growing body of literature indicates that social capital is critical for the psychosocial wellbeing in COVID-19 pandemic. A cross-sectional study of 170 self-isolated individuals in China has reported that a low level of social capital was associated with elevated risks of anxiety and stress. Moreover, a higher level of social capital was positively associated with the quality of sleep in those participants (Xiao et al., 2020). Moreover, in the United States, a higher social capital was found to be positively correlated with decreased mobility (Borgonovi and Andrieu, 2020), highlighting the reduction of community transmission, better self-sustenance, improved health outcomes, and associated psychosocial impacts. Similar studies suggest that social capital is negatively associated with COVID-19 growth rates and retail/recreational movements, and positively associated with residential movement (Varshney and Socher, 2020). This indicates that communities with high social capital may respond more efficaciously than those with low social capital (Pitas and Ehmer, 2020). Such responses may range from providing social support at the individual level to ensuring optimal social determinants of mental health in communities, which may subsequently result in a lower risk of mental health problems and improved mental wellbeing across populations.

\section{Setting global health priorities for enhancing social capital and mental health}

It is essential to adopt several strategies ensuring evidence-based practices to leverage social capital for improving mental health during COVID-19. First, the existing social capital and the burden of psychosocial problems in a community should be analyzed, which can be very much different across communities depending on psychosocial dynamics and how those are impacted by this pandemic. Such analyses would inform the community stakeholders, including mental health providers and social workers, to assess how social capital is organized in that community and what approaches may deploy them in promoting mental health and wellbeing. Secondly, mapping social and mental health resources may facilitate engaging those resources in crisis management and linking social capital and mental health in a community. Thirdly, social capital measures often target the social determinants of health through addressing unemployment, promoting education, and other means of empowering individuals and communities. As macroeconomic changes during 
COVID-19 are likely to affect individuals and communities, predominantly the marginalized ones, it is imperative to identify those vulnerable populations and prioritize such interventions for them. Fourth, psychosocially vulnerable individuals (for example, homeless people or those who have preexisting mental health problems) may have a higher need for psychosocial care, who might need integrated social capital interventions alongside specific care for mental health problems. Fifth, the use of digital media like the internet and social media is likely to influence the formation and utilization of social capital among individuals. As little research has been conducted on how social capital works within the digital ecosystem, a better understanding is needed to make informed use of such platforms aiming for psychosocial wellbeing. More research is needed in this domain to address the knowledge gap and use the same for improving mental health outcomes. Sixth, individuals may be exposed to misinformation and disinformation in social media that may affect mental health. It would be helpful if social networks are supported with such information that may reestablish trust among individuals and foster psychosocial solidarity (Tasnim et al., 2020). Lastly, systems-level measures like policies, programs, and proactive leadership would be needed to engage institutions and communities for effective development of social capital as well as mental health resilience in this pandemic.

\section{Conclusion and way forward}

Social capital can play critical roles in preventing mental health problems and promoting positive mental health across populations. With the growing psychosocial problems associated with COVID-19, communities and institutions are experiencing remarkable challenges to mitigate the consequences of this pandemic. In this scenario, the healthcare providers and policymakers may need to devise sustainable and evidence-based strategies minimizing the psychosocial impacts of this pandemic and promoting resilience across communities. Social capital research and practice may inform how public mental health can be strengthened using community resources. The ongoing approaches must incorporate strategic measures such as social capital interventions and evaluate how they work in different contexts, which may strengthen global psychosocial responses to the COVID-19 pandemic and future public health emergencies. 
Funding: No funding was received at any stage of preparing this article

Acknowledgement: None

Conflicts of interest: We declare no conflicts of interest

\section{References}

Aluh, D.O., Onu, J.U., 2020. The Need for Psychosocial Support Amid COVID-19 Crises in Nigeria. Psychol. Trauma Theory, Res. Pract. Policy 12. https://doi.org/10.1037/tra0000704

Borgonovi, F., Andrieu, E., 2020. Bowling together by bowling alone: Social capital and Covid19. Covid Econ. 17, 73-96.

Chen, H., Meng, T., 2015. Bonding, bridging, and linking social capital and self-rated health among Chinese adults: Use of the anchoring vignettes technique. PLoS One 10. https://doi.org/10.1371/journal.pone.0142300

De Silva, M.J., McKenzie, K., Harpham, T., Huttly, S.R.A., 2005. Social capital and mental illness: A systematic review. J. Epidemiol. Community Health 59, 619-627. https://doi.org/10.1136/jech.2004.029678

Ehsan, A.M., De Silva, M.J., 2015. Social capital and common mental disorder: A systematic review. J. Epidemiol. Community Health 69, 1021-1028. https://doi.org/10.1136/jech-2015205868

Ferlander, S., 2007. The importance of different forms of social capital for health. Acta Sociol. 50, 115-128. https://doi.org/10.1177/0001699307077654

Hossain, M.M., Sultana, A., Purohit, N., 2020a. Mental health outcomes of quarantine and isolation for infection prevention: A systematic umbrella review of the global evidence. Epidemiol. Health e2020038. https://doi.org/10.4178/epih.e2020038

Hossain, M.M., Tasnim, S., Sultana, A., Faizah, F., Mazumder, H., Zou, L., McKyer, E.L.J., Ahmed, H.U., Ma, P., 2020b. Epidemiology of mental health problems in COVID-19: a review. F1000Research 9, 636. https://doi.org/10.12688/f1000research.24457.1 
McPherson, K.E., Kerr, S., McGee, E., Morgan, A., Cheater, F.M., McLean, J., Egan, J., 2014. The association between social capital and mental health and behavioural problems in children and adolescents: an integrative systematic review. BMC Psychol. 2, 1-16. https://doi.org/10.1186/2050-7283-2-7

Noel, P., Cork, C., White, R.G., 2018. Social Capital and Mental Health in Post-Disaster/Conflict Contexts: A Systematic Review. Disaster Med. Public Health Prep. 12, 791-802. https://doi.org/10.1017/dmp.2017.147

Nyqvist, F., Forsman, A.K., Giuntoli, G., Cattan, M., 2013. Social capital as a resource for mental well-being in older people: A systematic review. Aging Ment. Heal. 17, 394-410. https://doi.org/10.1080/13607863.2012.742490

Pitas, N., Ehmer, C., 2020. Social Capital in the Response to COVID-19. Am. J. Heal. Promot. 089011712092453. https://doi.org/10.1177/0890117120924531

Rajkumar, R.P., 2020. COVID-19 and mental health: A review of the existing literature. Asian J. Psychiatr. 52, 102066. https://doi.org/10.1016/j.ajp.2020.102066

Sentell, T., Vamos, S., Okan, O., 2020. Interdisciplinary perspectives on health literacy research around the world: More important than ever in a time of covid-19. Int. J. Environ. Res. Public Health. https://doi.org/10.3390/ijerph17093010

Tasnim, S., Hossain, M.M., Mazumder, H., 2020. Impact of rumors or misinformation on coronavirus disease (COVID-19) in social media. J. Prev. Med. Public Heal. https://doi.org/10.3961/jpmph.20.094

Varshney, L.R., Socher, R., 2020. COVID-19 Growth Rate Decreases with Social Capital, medRxiv. Cold Spring Harbor Laboratory Press. https://doi.org/10.1101/2020.04.23.20077321

Xiao, H., Zhang, Y., Kong, D., Li, S., Yang, N., 2020. Social Capital and Sleep Quality in Individuals Who Self-Isolated for 14 Days During the Coronavirus Disease 2019 (COVID19) Outbreak in January 2020 in China. Med. Sci. Monit. Int. Med. J. Exp. Clin. Res. 26, e923921. https://doi.org/10.12659/MSM.923921 